\title{
Pharmacotherapies for fatigue in chronic liver disease (CLD): a systematic review and meta-analysis (protocol)
}

\author{
Andem Effiong ${ }^{1 *}$ (D) and Prerna Kumari ${ }^{2}$
}

\begin{abstract}
Background: This is the protocol for a systematic review (and meta-analysis) of an intervention. The primary objective of this systematic review will be to assess the benefits and harms of pharmacological therapies (pharmacotherapies) for the management of fatigue in adults with CLD of any etiology. The effects of pharmacological therapies on fatigue in CLD will be compared against those of placebo, no intervention, or non-pharmacological interventions. Specifically, this review will examine whether pharmacological therapies improve CLD-associated fatigue, and if they do, what key elements are associated with their effectiveness. The results of this systematic review will assist clinicians, policy-makers, researchers, and people with CLD in decision-making on how best to manage fatigue and its associated symptoms.

Methods: MEDLINE, SCOPUS, EMBASE, EU Clinical Trials Register, WHO International Clinical Trials Registry Platform, CENTRAL (The Cochrane Library), ClinicalTrials.gov, reference lists of articles and conference proceedings will be searched for relevant studies. No language or date restrictions will be applied. Eligible studies will include adults with CLD of any etiology. Included studies will be randomized controlled trials. From included studies, data on participant characteristics, study design, setting, research ethics compliance, and intervention outcomes will be extracted. Risk of bias in included studies will be assessed using the Cochrane Risk of Bias Tool. A random-effects meta-analysis will be conducted. If substantial or considerable levels of heterogeneity are detected, analysis will be limited to a narrative synthesis.

Discussion: This systematic review will examine the effectiveness of pharmacological therapies on fatigue reduction in people with CLD. Such therapies may be more effective than non-pharmacological interventions in treating fatigue symptoms in CLD. Evidence derived from the findings of this study will guide future practice, policy, and research.
\end{abstract}

Systematic review registration: PROSPERO, CRD42017076957

Keywords: Fatigue, Liver disease

\section{Background}

For the purposes of this systematic review, we define chronic liver disease (CLD) as progressive destruction of normal liver functions, commonly associated with fibrotic regeneration of the liver tissue [1]. Diseases which comprise CLD include compensated cirrhosis of any etiology, chronic hepatitis, alcoholic liver disease (ALD), non-alcoholic steatohepatitis (NASH), cholestatic liver disease, and metabolic liver disease. Major underlying causes of CLD vary depending on geographical location.

\footnotetext{
* Correspondence: andem.effiong@me.com

'Georgetown University, 3700 O St NW, Washington, DC 20057, USA

Full list of author information is available at the end of the article
}

In low- and middle-income countries, etiologies for CLD are predominantly viral, while in western countries and some regions of the Indian subcontinent, alcohol is frequently implicated $[2,3]$. In the USA, non-alcoholic fatty liver disease (NAFLD), a form of NASH, and defined as abnormal accumulation ( $>5 \%$ ) of hepatic triglyceride in the absence of excess alcohol consumption, is the most common form of CLD [4]. Because CLD encompasses a broad spectrum of hepatic pathology, different diagnostic tools for screening exist. Diagnostic tools may be invasive (e.g., liver biopsy) or non-invasive (e.g., biochemical markers and imaging techniques) $[4,5]$. This review will include studies in which CLD was detected 
and confirmed with at least one of the following diagnostic tools: laboratory examination, liver biopsy, or radiologic imaging.

Worldwide, CLD is a major contributor of morbidity and mortality and therefore constitutes a significant public health burden [6, 7]. Clinical signs of CLD include jaundice, ascites, gynecomastia, encephalopathy, and spider angioma; however, these signs are not necessarily specific for CLD, and their presence in people with risk factors or other indicators of liver disease may require management strategies encompassing a well-structured liver disease workup to determine their etiology [8].

Fatigue may be acute (self-limiting) or chronic (debilitating and of greater than 6 months duration [9]. Fatigue is a multidimensional symptom often experienced as loss of energy and resulting in disparities between intention and ability. Abnormalities of circadian rhythm, e.g., increased daytime somnolence, have been linked to the pathogenesis of fatigue [10, 11]. Increased central serotoninergic tone is also believed to be a precursor of fatigue in chronic illness. Among patients with CLD, even though fatigue is the most prevalent symptom, its pathogenesis is not well understood. Moreover, the overlap of fatigue in CLD with other neuropsychiatric conditions, such as depression and anxiety, makes objective interpretation and treatment extremely difficult [10, 11]. In CLD, fatigue is thought to originate from the central nervous system (CNS) and is often marked by a reduction in quality of life, partly attributed to challenges in completing daily life activities [11]. CLD patients with decompensated cirrhosis or liver failure may also suffer from peripheral fatigue (characterized by muscle wasting and weakness) as a result of neuromuscular dysfunction. It is worth noting that both peripheral and central fatigue can occur simultaneously [12].

Few pharmacological or drug interventions currently exist primarily for the treatment of symptoms associated with fatigue in people with CLD [13]. Some nonpharmacological interventions, e.g., psychosocial interventions, physical activity, and lifestyle modifications have been found to be effective in reducing fatigue or promoting improved liver function in liver disease; however, their results have been mixed. Although fatigue in CLD is pervasive, there is no established therapy for its management [14]. Pharmacological interventions targeting fatigue reduction in people living with CLD are classified as either specific or non-specific therapies [14]. Accordingly, therapeutic effects of both specific and non-specific interventions will be examined.

Due to the complexities involved in objectively measuring fatigue, assessments of fatigue in people with CLD are usually done with questionnaires (e.g., Short Form-36 (SF-36), Fatigue Severity Scale (FSS), or Fatigue Impact Scale (FIS)) which permit objective quantification or scoring based on an individual's answers to a particular set of questions regarding fatigue $[15,16]$. Alterations in fatigue scores are therefore used to ascertain whether therapeutic interventions facilitate improvement or worsening of feelings or symptoms of fatigue $[15,16]$.

People with CLD who suffer from frequent fatigue often have numerous functional impairments and lower quality of life $[17,18]$. Nonetheless, their experiences and symptoms are often underreported and undertreated $[17,18]$. The lack of clarity and dearth of evidence on the effects of pharmacological therapies for the management of fatigue in CLD makes it imperative to conduct a systematic review which further investigates the benefits and harms of these therapies, especially among those who suffer from severe fatigue $[19,20]$.

\section{Methods/design}

This systematic review protocol was developed in accordance with the Preferred Reporting Items of Systematic Reviews and Meta-Analysis for Protocols (PRISMAP) 2015 [21]. The completed PRISMA-P checklist is included as an additional file (see Additional file 1). The PRISMA extension for Abstracts (PRISMA-A) was used as a checklist to develop the protocol abstract [22]. This 12-item checklist will also be used as a framework to develop the abstract of the systematic review. In the event of any amendments to this protocol, the date of each amendment and its justification will be updated in the registration record and completed systematic review. The systematic review will adhere to the reporting guidelines of the Preferred Reporting Items for Systematic Reviews and Meta-Analyses (PRISMA) statement [23]. Its methodological quality will be appraised and reported using A Measurement Tool to Assess Systematic Reviews (AMSTAR) 2 [24].

\section{Objectives}

The primary objective of this systematic review will be to assess the benefits and harms of pharmacological therapies (pharmacotherapies) for the management of fatigue in adults with CLD of any etiology. Specifically, this review will examine whether pharmacological therapies improve CLD-associated fatigue, and if they do, what key elements are associated with their effectiveness.

\section{Review question}

Are pharmacological interventions alone or in combination with other pharmacological interventions more effective than placebo, no intervention, or non-pharmacological interventions in the management of fatigue in people with CLD? 


\section{Eligibility criteria \\ Study design}

Studies selected for inclusion in this systematic review will be limited to randomized controlled trials. Individual studies must have assessed fatigue as a primary or secondary outcome measure, and not solely as an adverse effect, with results reported independently for CLD. Quasirandomized controlled trials will be excluded.

\section{Participants/population}

Eligible populations will include adults with all types of CLD, regardless of severity or time since diagnosis of illness.

\section{Interventions}

This review will include pharmacological interventions used in the management of fatigue in CLD. Interventions will be included irrespective of delivery setting or person delivering the intervention. Pharmacological interventions will be compared either against each other or placebo (or no intervention) or non-pharmacological interventions.

\section{Comparator}

- Placebo (or no intervention)

- Other pharmacological interventions

- Non-pharmacological interventions

\section{Outcomes}

Outcome measurements will be assessed at baseline and post-treatment at different lengths of follow-up. Fatigue will encompass malaise, lethargy, exhaustion, and decreased vitality [10].

\section{○ Primary outcomes}

- FSS score (lower limit = 1; upper limit = 7; final score = mean value of the 9 items; with lower scores being favorable, i.e., FSS score $\geq 4$ being indicative of fatigue) [20].

- Secondary outcomes

- Fatigue scores (other, validated)

- Quality of life

- Adverse events (including all causes of mortality)

\section{Search strategy}

An extensive search of the literature will be conducted in the following databases:

MEDLINE, SCOPUS, EMBASE, Clinicaltrials.gov, EU Clinical Trials Register, WHO International Clinical Trials Registry Platform, and CENTRAL (The Cochrane Library). There will be no date or language limits. Reference lists of eligible studies and conference proceedings will also be searched. The provisional search strategies for MEDLINE and EMBASE are included as an additional file (see Additional file 2).

\section{Study selection and screening}

Titles and abstracts of all potentially eligible studies will be retrieved and screened by both $\mathrm{AE}$ and PK. And then, they will both independently screen full texts of studies (that were selected during the initial title and abstract screening) for inclusion in the systematic review.

Any initial disagreements on study selection will be resolved through discussion. In the event that AE and PK fail to agree on study eligibility, a non-author colleague designated a priori as an arbiter will be invited to make a final decision.

\section{Data extraction and management}

$\mathrm{AE}$ and PK will independently appraise and extract data using a standardized data abstraction form. The data extraction process for each included study will be guided by AMSTAR 2 recommendations [24] and will include the following information:

- Design, sponsor, conflict of interest statement, aims and objectives, risk of bias, demographic characteristics, and outcomes assessed;

- Number of participants randomized for each study arm, type of placebo, non-pharmacological intervention or pharmacological intervention used in comparator arm, type of pharmacological therapy used in experimental arm, what amounts to no intervention when applicable;

- Doses administered, clinical outcomes (e.g., FSS scores, quality of life, adverse events), and surrogate outcomes (e.g., non-adherence and missing data, i.e., attrition or loss to follow-up).

To minimize the likelihood of selective outcome reporting, all time-points measured for each outcome within each study will be reported [25]. Study investigators will be contacted via email for missing data or confirmation of available data.

\section{Methodological quality}

AMSTAR 2 will be used to assess the methodological quality of the systematic review.

AMSTAR 2 is a validated 16-item instrument that is used to critically appraise systematic reviews of randomized controlled trials [24].

\section{Risk of bias assessment}

The Cochrane Risk of Bias Tool which covers six domains, namely, selection bias (random sequence generation and allocation concealment), performance bias (blinding of participants and personnel), detection bias 
(blinding of outcome assessment), attrition bias (incomplete outcome data), reporting bias (selective reporting), and other bias (other potential sources of bias) will be used to assess risk of bias for each included trial [26]. The sequence generation process described by the investigators will be assessed to determine whether it was truly random.

For each domain, a judgment of high risk of bias, low risk of bias, or unclear risk of bias will be assigned [26]. Risk of bias will be assessed regardless of any expected variability in study results or validity.

\section{Quality of the evidence}

The quality of evidence obtained will be assessed using the Grading of Recommendations Assessment, Development and Evaluation (GRADE) approach [27]. AE and PK will individually assess the quality of the evidence. Disagreements will be resolved through arbitration by a non-author colleague.

\section{Data synthesis and analysis}

Data will be analyzed using the Cochrane Review Manager (RevMan) 5.3 [28]. We will use a random-effects model for meta-analysis if moderate heterogeneity is detected [29]. Where substantial or considerable levels of heterogeneity are detected, we will limit our analysis to a narrative synthesis [29].

\section{Measuring treatment effect}

Risk ratio or relative risk (RR) will be used as summary statistics for dichotomous outcomes. For continuous data, we will use the mean difference (MD) or standardized mean difference (SMD) as summary statistics calculated as single assessments or change from baseline measures of individual data [30]. If possible, we will summarize time-to-event data by survival analysis 152 and present the intervention effect as a hazard ratio (HR) [25]. Or, we may analyze time-to-event data as dichotomous data if the status for all patients within a study is presented at a fixed time point, and a HR analysis is not feasible [30]. We will present all measures of effect with 95\% confidence intervals (CI) [30].

\section{Units of analysis issues}

We will take into account the level at which each randomization occurred. If appropriate, we will consider whether each participant in a trial was subject to more than one intervention, or whether there were different observations for the same outcome, by evenly distributing the comparator and experimental group participants evenly between both groups. Available data from all randomized controlled trial phases will be assessed for suitability for inclusion in the analysis.

\section{Missing data}

Should we encounter missing data, we will attempt to contact study investigators or sponsors to obtain numerical outcome data or validate important study attributes. Where possible, we will conduct analyses based on the intention-to-treat (ITT) principle [30]. If there is loss to follow-up participants, missing outcomes will be excluded from the analysis.

\section{Assessment of heterogeneity}

Heterogeneity across all the studies will be explored by using a combination of these measures:

I. Examination of forest plots and their associated outputs, e.g., Cochran's Q

II. Determination of $I^{2}$ statistic, i.e., variation in effect size due to heterogeneity (with $30-59 \%, 60-89 \%$, and $90-100 \%$ representing moderate, substantial, and considerable levels of heterogeneity)

III.Determination of $T^{2}$ and tau (i.e., estimate of between-study variance or measure of the dispersion of true effect sizes between studies based on the magnitude of the effect size) for determining the prediction interval.

\section{Subgroup analysis}

If appropriate, we will further investigate sources of heterogeneity through analyses by the following subgroups:

I. Age

II. Sex/gender

III.Race/ethnicity

IV.Education

V. Type of CLD

Potential confounders of subgroup effects that we may consider include the following:

- Age

- Comorbidities (e.g., depression, anxiety, post viral infection, unspecified somatoform disorder, chronic fatigue syndrome, thyroid disorder, and anemia)

- Intervention features: intensity, content, delivery

- Length of study

An in-depth analysis of each subgroup will be done by stratification of the effect modifying variable [31]. We will assume that risks are unevenly distributed. To overcome the effects of confounders, we will first adjust for confounders in our statistical analyses within relevant subgroups to quantify their effect size followed by an assessment within subgroups that are stratified by the effect modifying variable [31-33]. 
Where stratification becomes impractical, we will conduct a multivariate regression [32, 33].

To determine whether perceived differences between subgroups are clinically relevant, we will calculate effect sizes (Cohen's $d$ ) and correlations for the main variables, and make comparisons between initial and final values derived from the original sample and subgroups. Determination of the robustness of observed effects will be guided partly by the degree of consistency of our primary outcome (fatigue) analysis over different subgroups. Lastly, we will report on whether each subgroup variable assessed was measured at baseline or after randomization because measurements after randomization may be less credible.

\section{Sensitivity analysis}

If appropriate, we will conduct a sensitivity analysis to test the robustness of our findings. We will evaluate the effects of methodological quality on the pooled estimate by repeating the analysis after excluding trials that were deemed high or unclear risk within at least two domains, namely, performance and detection bias [25].

\section{Assessment of publication bias}

Where possible, reporting and publication bias will be assessed by way of funnel plots created to identify asymmetry [30]. The choice of test for funnel plot asymmetry will be dependent of the degree of heterogeneity observed [30].

\section{Discussion}

This systematic review will examine and synthesize the evidence of the benefits and harms of pharmacological interventions in the management of fatigue in people with CLD of any etiology. Currently, few high quality studies exist that have evaluated the use of pharmacotherapies in the treatment of fatigue in people with CLD.

Such therapies may be more effective than nonpharmacological interventions in treating fatigue symptoms in CLD. Evidence derived from the findings of this study will guide future practice, policy, and research. Study findings will also be disseminated to target audiences in different settings and published in a peer reviewed journal.

\section{Additional files}

Additional file 1: PRISMA-P CHECKLIST. (DOCX $28 \mathrm{~kb})$

Additional file 2: Search strategy <MEDLINE \& EMBASE $>$. (DOCX $11 \mathrm{~kb}$ )

\section{Abbreviations}

ALD: Alcoholic liver disease; AMSTAR: A Measurement Tool for the Assessment of Multiple Systematic Reviews; Cl: Confidence interval; CLD: Chronic liver disease; EU: European Union; FIS: Fatigue Impact Scale; FSS: Fatigue Severity Scale; GRADE: Grading of Recommendations Assessment, Development and Evaluation; HR: Hazard ratio; MD: Mean difference; NAFLD: Non-alcoholic fatty liver disease; NASH: Non-alcoholic steatohepatitis; PBC: Primary biliary cholangitis; PRISMA: Preferred Reporting Items for Systematic Reviews and Meta-Analyses; PRISMA-P: Preferred Reporting Items for Systematic Reviews and Meta-Analysis Protocols; RevMan: Review Manager; RR: Risk ratio or relative risk; SMD: Standardized mean difference; WHO: World Health Organization

\section{Acknowledgements}

We would like to thank Ms. Martina Darragh of Georgetown University for assisting with the search strategy.

\section{Funding}

No funding was received throughout the duration of the study.

Availability of data and materials

Not applicable.

Authors' contributions

AE and PK conceived the idea for the protocol, systematic review, and meta-analysis. They are both guarantors of the review. All authors read and approved the final manuscript.

Ethics approval and consent to participate

Not applicable.

Consent for publication

Not applicable.

Competing interests

The authors declare that they have no competing interests.

\section{Publisher's Note}

Springer Nature remains neutral with regard to jurisdictional claims in published maps and institutional affiliations.

\section{Author details}

${ }^{1}$ Georgetown University, 3700 O St NW, Washington, DC 20057, USA.

${ }^{2}$ Manipal College of Pharmaceutical Sciences, Manipal, Karnataka, India.

Received: 22 September 2017 Accepted: 15 January 2018

Published online: 14 February 2018

\section{References}

1. Martí-Carvajal AJ, Solá I. Vitamin K for upper gastrointestinal bleeding in people with acute or chronic liver diseases. Cochrane Database Syst Rev. 2015;Issue 6:Art. No.: CD004792. https://doi.org/10.1002/14651858.CD004792. pub5

2. Abbas Z, Shazi L. Pattern and profile of chronic liver disease in acute on chronic liver failure. Hepatol Int. 2015;9(3):366-72. https://doi.org/10.1007/ s12072-015-9627-z. Epub 2015 May 28

3. Mahtab MA, Rahman S, Khan M, Karim MF. Hepatitis E virus is a leading cause of acute-on-chronic liver disease: experience from a tertiary centre in Bangladesh. Hepatobiliary Pancreat Dis Int. 2009;8:50-2.

4. Nalbantoglu IL, Brunt EM. Role of liver biopsy in nonalcoholic fatty liver disease. World J Gastroenterol. 2014;20(27):9026-37. https://doi.org/10.3748/ wjg.v20.i27.9026.

5. Tsuchiya N, Sawada Y, Endo I, Saito K, Uemura Y, Nakatsura T. Biomarkers for the early diagnosis of hepatocellular carcinoma. World J Gastroenterol. 2015; 21(37):10573-83. https://doi.org/10.3748/wjg.v21.i37.10573.

6. Low J, Davis S, Vickerstaff V, Greenslade L, Hopkins K, Langford A, et al. Advanced chronic liver disease in the last year of life: a mixed methods study to understand how care in a specialist liver unit can be improved. BMJ Open. 2017;7(8):e016887. https://doi.org/10.1136/bmjopen-2017016887

7. Sepanlou SG, Malekzadeh F, Delavari F, Naghavi M, Forouzanfar MH, MoradiLakeh $\mathrm{M}$, et al. Burden of gastrointestinal and liver diseases in Middle East and North Africa: results of global burden of diseases study from 1990 to 2010. Middle East J Dig Dis. 2015;7(4):201-15.

8. Oeda S, Takahashi H, Ogawa Y, Imajo K, Yoneda M, et al. Prevalence of pruritus in patients with chronic liver disease: a multicenter study. Hepatol Res. 2017; https://doi.org/10.1111/hepr.12978. 
9. Golabi P, Sayiner M, Bush H, Gerber LH, Younossi ZM. Patient-reported outcomes and fatigue in patients with chronic hepatitis $\mathrm{C}$ infection. Clin Liver Dis. 2017;21(3):565-78. https://doi.org/10.1016/j.cld.2017.03.011.

10. Swain MG. Fatigue in liver disease: pathophysiology and clinical management. Can J Gastroenterol. 2006;20(3):181-8.

11. Kallman J, O'Neill MM, Larive B, Boparai N, Calabrese L, Younossi ZM. Fatique and health-related quality of life (HRQL) in chronic hepatitis $C$ virus infection. Dig Dis Sci. 2007:52(10):2531-9.1.

12. Weinstein AA, Diao G, Baghi H, Escheik C, Gerber LH, Younossi ZM. Demonstration of two types of fatigue in subjects with chronic liver disease using factor analysis. Qual Life Res. 2017;26(7):1777-84. https://doi.org/10. 1007/s11136-017-1516-6.

13. Mücke M, Mochamat $\mathrm{CH}$, Peuckmann-Post V, Minton O, Stone P, Radbruch L. Pharmacological treatments for fatigue associated with palliative care. Cochrane Database Syst Rev. 2015;5:CD006788. https://doi.org/10.1002/ 14651858.CD006788.pub3.

14. Kaser S, Ebenbichler CF, Tilg H. Pharmacological and non- pharmacological treatment of non-alcoholic fatty liver disease. Int J Clin Pract. 2010;64(7): 968-83. https://doi.org/10.1111/j.1742- 1241.2009.02327.x. Review. PMID: 20584230

15. Neuberger GB. Measures of fatigue. Arthritis \& Rheumatism (Arthritis Care \& Research). 2003;49(5S):15: S175- S183. https://doi.org/10.1002/art.11405.

16. Schwartz JE, Jandorf L, Krupp LB. The measurement of fatigue: a new instrument. J Psychosom Res. 1993;37(7):753-62.

17. Newton JL. Fatigue in primary biliary cirrhosis. Clin Liver Dis. 2008;12(2):36783; ix. https://doi.org/10.1016/j.cld.2008.02.010.

18. Ghabril M, Jackson M, Gotur R, Weber R, Orman E, Vuppalanchi R, et al. Most individuals with advanced cirrhosis have sleep disturbances, which are associated with poor quality of life. Clin Gastroenterol Hepatol. 2017;15(8): 1271-1278.e6. https://doi.org/10.1016/j.cgh.2017.01.027.

19. Silveira MG, Gossard AA, Stahler AC, Jorgensen RA, Petz JL, Ali AH, et al. A randomized, placebo-controlled clinical trial of efficacy and safety: Modafinil in the treatment of fatigue in patients with primary biliary cirrhosis. Am J Ther. 2017;24(2):e167-76. https://doi.org/10.1097/MJT.0000000000000387.

20. Hjollund $\mathrm{NH}$. Assessment of fatigue in chronic disease: a bibliographic study of fatigue measurement scales. Health Qual Life Outcomes. 2007;5:12.

21. Shamseer $L$, Moher D, Clarke M, Ghersi D, Liberati A, Petticrew M, Shekelle P, Stewart LA, PRISMA-P Group. Preferred reporting items for systematic review and meta-analysis protocols (PRISMA-P) 2015: elaboration and explanation. BMJ. 2015;350:g7647. https://doi.org/10.1136/bmj.g7647. Erratum in: BMJ. 2016;354:14086

22. Beller EM, Glasziou PP, Altman DG, et al. PRISMA for abstracts: reporting systematic reviews in journal and conference abstracts. PLoS Med. 2013; 10(4):e1001419. https://doi.org/10.1371/journal.pmed.1001419.

23. Moher D, Shamseer L, Clarke M, Ghersi D, Liberati A, Petticrew M, Shekelle P, Stewart LA, PRISMA-P Group. Preferred reporting items for systematic review and meta-analysis protocols (PRISMA-P) 2015 statement. Syst Rev. 2015;4:1. https://doi.org/10.1186/2046-4053-4-1.

24. Shea BJ, Reeves BC, Wells G, Thuki M, Hamel C, Moran J, et al. AMSTAR 2: a critical appraisal tool for systematic reviews that include randomised or non-randomised studies of healthcare interventions, or both. BMJ. 2017;358: j4008. https://doi.org/10.1136/bmj.j4008

25. Page MJ, McKenzie JE, Kirkham J, Dwan K, Kramer S, Green S, et al. Bias due to selective inclusion and reporting of outcomes and analyses in systematic reviews of randomized trials of healthcare interventions. Cochrane Database Syst Rev. 2014;10:MR000035. https://doi.org/10.10002/14651858.MR000035. pub2.

26. Higgins JP, Altman DG, Gøtzsche PC, Jüni P, Moher D, Oxman AD, et al. The Cochrane Collaboration's tool for assessing risk of bias in randomised trials. BMJ. 2011;343:d5928. https://doi.org/10.1136/bmj.d5928.

27. Guyatt GH, Oxman AD, Vist GE, Kunz R, Falck-Ytter Y, Alonso- Coello P, et al. GRADE: an emerging consensus on rating quality of evidence and strength of recommendations. BMJ. 2008;336(7650):924-6. https://doi.org/10.1136/ bmj.39489.470347.AD.

28. Review Manager (RevMan). [Computer program]. Version 5.3. Copenhagen: The Nordic Cochrane Centre, The Cochrane Collaboration; 2014

29. Deeks JJ, Higgins JPT, Altman DG (editors). Chapter 9: analysing data and undertaking meta-analyses. In: Higgins JPT, Green S (editors). Cochrane handbook for systematic reviews of interventions version 5.1.0 (updated march 2011). The Cochrane Collaboration, 2011. Available from http:// handbook-5-1.cochrane.org/.
30. Schünemann HJ, Oxman AD, Vist GE, Higgins JPT, Deeks JJ, Glasziou P, Guyatt GH. Chapter 12: interpreting results and drawing conclusions. In: Higgins JPT, Green S (editors), Cochrane handbook for systematic reviews of interventions version 5.1.0 (updated march 2011). The Cochrane Collaboration, 2011. Available from http://handbook-5-1.cochrane.org/.

31. Tanniou T, van der Tweel I, Teerenstra S, Roes KCB. Subgroup analyses in confirmatory clinical trials: time to be specific about their purposes. BMC Med Res Methodol. 2016;16:20. Published online 2016. https://doi.org/10. 1186/s12874-016-0122-6.

32. Whitlock EP, Eder M, Thompson JH, Jonas DE, Evans CV, Guirguis-Blake JM, Whitlock EP, Eder $M$, Thompson JH, et al. An approach to addressing subpopulation considerations in systematic reviews: the experience of reviewers supporting the U.S. preventive services task force. Systematic Reviews. 2017;6:41. https://doi.org/10.1186/s13643-017-0437-3.

33. Knol MJ, VanderWeele TJ. Recommendations for presenting analyses of effect modification and interaction. Int J Epidemiol. 2012;41(2):514-20. https://doi.org/10.1093/ije/dyr218. Epub 2012 Jan 9

\section{Submit your next manuscript to BioMed Central and we will help you at every step:}

- We accept pre-submission inquiries

- Our selector tool helps you to find the most relevant journal

- We provide round the clock customer support

- Convenient online submission

- Thorough peer review

- Inclusion in PubMed and all major indexing services

- Maximum visibility for your research

Submit your manuscript at www.biomedcentral.com/submit
) Biomed Central 